University of Nebraska - Lincoln

DigitalCommons@University of Nebraska - Lincoln

U.S. Department of Veterans Affairs Staff

Publications

U.S. Department of Veterans Affairs

$9-2005$

\title{
Vagus Nerve Stimulation for Treatment-Resistant Depression: A Randomized, Controlled Acute Phase Trial
}

\author{
A. John Rush \\ University of Texas Southwestern Medical Center, John.Rush@UTSouthwestern.edu \\ Lauren B. Marangell \\ Baylor College of Medicine \\ Harold A. Sackeim \\ New York State Psychiatric Institute, has1@Columbia.EDU \\ Mark S. George \\ Medical University of South Carolina, georgem@musc.edu \\ Stephen K. Brannan \\ Cyberonics Inc. \\ See next page for additional authors
}

Follow this and additional works at: https://digitalcommons.unl.edu/veterans

Rush, A. John; Marangell, Lauren B.; Sackeim, Harold A.; George, Mark S.; Brannan, Stephen K.; Davis, Sonia M.; Howland, Robert; Kling, Mitchel A.; Rittberg, Barry; Burke, William J.; Rapaport, Mark H.; Zajecka, John; Nierenberg, Andrew A.; Husain, Mustafa M.; Ginsberg, David; and Cooke, Robert G., "Vagus Nerve Stimulation for Treatment-Resistant Depression: A Randomized, Controlled Acute Phase Trial" (2005). U.S. Department of Veterans Affairs Staff Publications. 70.

https://digitalcommons.unl.edu/veterans/70

This Article is brought to you for free and open access by the U.S. Department of Veterans Affairs at DigitalCommons@University of Nebraska - Lincoln. It has been accepted for inclusion in U.S. Department of Veterans Affairs Staff Publications by an authorized administrator of DigitalCommons@University of Nebraska - Lincoln. 


\section{Authors}

A. John Rush, Lauren B. Marangell, Harold A. Sackeim, Mark S. George, Stephen K. Brannan, Sonia M. Davis, Robert Howland, Mitchel A. Kling, Barry Rittberg, William J. Burke, Mark H. Rapaport, John Zajecka, Andrew A. Nierenberg, Mustafa M. Husain, David Ginsberg, and Robert G. Cooke 


\title{
Vagus Nerve Stimulation for Treatment-Resistant Depression: A Randomized, Controlled Acute Phase Trial
}

\author{
A. John Rush, Lauren B. Marangell, Harold A. Sackeim, Mark S. George, Stephen K. Brannan, Sonia M. Davis, \\ Robert Howland, Mitchel A. Kling, Barry R. Rittberg, William J. Burke, Mark H. Rapaport, John Zajecka, \\ Andrew A. Nierenberg, Mustafa M. Husain, David Ginsberg, and Robert G. Cooke
}

\begin{abstract}
Background: Vagus nerve stimulation (VNS) alters both concentrations of neurotransmitters or their metabolites and functional activity of central nervous system regions dysregulated in mood disorders. An open trial has suggested efficacy.

Methods: This 10-week, acute, randomized, controlled, masked trial compared adjunctive VNS with sham treatment in 235 outpatients with nonpsychotic major depressive disorder $(\mathrm{n}=210)$ or nonpsychotic, depressed phase, bipolar disorder $(\mathrm{n}=25)$. In the current episode, participants had not responded adequately to between two and six research-qualified medication trials. A two-week, single-blind recovery period (no stimulation) and then 10 weeks of masked active or sham VNS followed implantation. Medications were kept stable. Primary efficacy outcome among 222 evaluable participants was based on response rates ( $\geq 50 \%$ reduction from baseline on the 24-item Hamilton Rating Scale for Depression [HRSD 24$]$ ).

Results: At 10-weeks, HRSD ${ }_{24}$ response rates were $15.2 \%$ for the active $(\mathrm{n}=112)$ and $10.0 \%$ for the sham $(\mathrm{n}=110) \mathrm{groups}(\mathrm{p}=.251$, last observation carried forward [LOCF]). Response rates with a secondary outcome, the Inventory of Depressive SymptomatologySelf-Report (IDS-SR 30 ), were $17.0 \%$ (active) and 7.3\% (sham) ( $\mathrm{p}=.032$, LOCF). VNS was well tolerated; 1\% (3/235) left the study because of adverse events.
\end{abstract}

Conclusions: This study did not yield definitive evidence of short-term efficacy for adjunctive VNS in treatment-resistant depression.

Key Words: Vagus nerve stimulation (VNS), major depressive disorder, bipolar disorder, treatment-resistant depression (TRD), clinical trial, efficacy, side effects

$\mathrm{T}$ he Vagus Nerve Stimulation (VNS) Therapy System has been shown to reduce the frequency of seizures among patients with pharmacoresistant epilepsy (Ben-Menachem et al 1994; Handforth et al 1998; Vagus Nerve Stimulation Study Group 1995). Over 29,000 patients with epilepsy

From the Department of Psychiatry (AJR, MMH), University of Texas Southwestern Medical Center, Dallas; Department of Psychiatry (LBM), Baylor College of Medicine, South Central Mental IIIness Research Educational and Clinical Center, Houston; Cyberonics, Inc. (SKB), Houston, Texas; Department of Biological Psychiatry (HAS), New York State Psychiatric Institute, New York; Departments of Psychiatry and Radiology (HAS), College of Physicians and Surgeons of Columbia University, New York; Department of Psychiatry (DG), New York University Medical Center, New York, New York; Department of Psychiatry (MSG), Medical University of South Carolina, Charleston; Ralph H. Johnson Veterans Hospital (MSG), Charleston, South Carolina; Quintiles Inc. (SMD), Research Triangle Park, North Carolina; Department of Psychiatry (RH), University of Pittsburgh School of Medicine, Pittsburgh, Pennsylvania; Department of Veterans Affairs/ Mental Health Service Line and Department of Psychiatry (MAK), University of Maryland School of Medicine, Baltimore, Maryland; Department of Psychiatry (BRR), University of Minnesota Medical School - Riverside, Minneapolis, Minnesota; Department of Psychiatry (WJB), University of Nebraska Medical Center, Omaha, Nebraska; Department of Psychiatry (MHR), University of California at San Diego Medical Center, La Jolla, California; Department of Psychiatry (JZ), Rush-Presbyterian-St. Luke's Medical Center, Chicago, Illinois; Department of Psychiatry (AAN), Massachusetts General Hospital, Boston, Massachusetts; Department of Psychiatry (RGC), University of Toronto, Toronto, Canada.

Address reprint requests to A. John Rush, M.D., University of Texas Southwestern Medical Center, 5323 Harry Hines Boulevard, Dallas, TX 753909086; E-mail: John.Rush@UTSouthwestern.edu.

Received November 30, 2004; revised A pril 20, 2005; accepted May 13, 2005. have been implanted with the VNS device (data on file with Cyberonics), and few clinically significant adverse events (AEs) have been noted (Schachter and Saper 1998).

The rationale for evaluating the antidepressant efficacy of VNS has been reviewed elsewhere (George et al 2000; Rush et al 2000b). In brief, VNS alters the cerebrospinal fluid (CSF) concentrations of neurotransmitters or their metabolites (e.g., increased gamma-aminobutyric acid (GABA), increased 5-hydroxyindoleacetic acid (5H1AA), increased homovanillic acid (Carpenter et al 2004; Hammond et al 1992; Ben-Menachem et al 1995) in animal and human studies. VNS also alters the functional activity of CNS regions, (e.g., orbital frontal cortex, insula, thalamus, hypothalamus, cingulate, and hippocampus) dysregulated in mood disorders (Chae et al 2003; Henry et al 1998). Third, certain anticonvulsants (and, therefore, potentially VNS) have therapeutic value in mood disorders. Finally, epilepsy patients who have received VNS have shown improvement in depressive symptoms independent of the degree of seizure control (Elger et al 2000; Harden et al 2000).

A 10-week acute phase, open trial (Sackeim et al 2001b) found a $30.5 \%$ response rate (i.e., $\geq 50 \%$ reduction in $\mathrm{HRSD}_{28}$ scores) among outpatients $(n=59)$ with treatment-resistant, nonpsychotic major depressive episodes (MDEs). Naturalistic follow-up of these 59 patients revealed $44 \%$ response and $27 \%$ remission rates (LOCF) after 1 year of VNS, and 44\% response and $22 \%$ remission rates (LOCF) after 2 years of VNS (Rush et al 2003a). Given the severity of the mood disorder and the degree of prior treatment resistance of the patients in the pilot study, the acute and 1-year follow-up findings were seen as supporting the possibility that VNS has clinically important antidepressant effects, which formed the basis for this double-masked, randomized, sham-controlled acute trial. Specifically, we hypothesized that 10 weeks of VNS, as opposed to 10 weeks of sham VNS, would result in a greater proportion of responders based on $\mathrm{a} \geq 50 \%$ reduction in baseline $\mathrm{HRSD}_{24}$. 


\section{Methods and Materials}

\section{Participants}

To participate in this study, participants had to have a current DSM-IV (American Psychiatric Association 2000) primary diagnosis of major depressive disorder (MDD) or bipolar I or II disorder (BPI or BPII). To ensure that the participants in the study required long-term treatment, entrance criteria required that participants be in the current major depressive episode (MDE) for $\geq 2$ years or to have had at least four lifetime major depressive episodes, including their current MDE. The study included men or women, aged 18 to 80 years. Women could not be pregnant and had to use acceptable birth control methods (including abstinence). The baseline score of the $\mathrm{HRSD}_{24}$ (average of two measures) had to be $\geq 20$. Participants with bipolar disorder had to be resistant to, intolerant of, or have a medical contraindication to lithium.

Exclusionary criteria included atypical or psychotic features in any MDE; lifetime history of any nonmood psychotic disorder (e.g., schizophrenia); current rapid cycling bipolar disorder; or a current secondary diagnosis of delirium, dementia, amnesia, or other cognitive disorder (based on DSM-IV criteria). Participants with clinically significant current suicidal intent and those with certain risks related to the surgical implantation of the VNS device were also excluded.

Treatment resistance was defined for the current MDE using a modified Antidepressant Treatment History Form (ATHF) (Prudic et al 1990, 1996; Sackeim 2001; Sackeim et al 1990). For the current MDE, participants must have had an unsatisfactory response to at least two adequate trials of different classes of antidepressant medication, but not more than six, regardless of antidepressant category based on participant/family interviews, medical records, and, when available, pharmacy records. Trial adequacy was defined by both the ATHF dose and duration criteria for each medication trial. Each medication trial was scored on a scale of 1 to 4 according to the Antidepressant Resistance Rating (ARR) scale. An ARR score of $\geq 3$ was required to designate a trial as adequate. For example, acceptable trials of fluoxetine or imipramine required at least 4 weeks of treatment at a minimum dose of 20 or $200 \mathrm{mg} /$ day, respectively. Medications that could potentially receive a rating of 3 or higher, indicating an adequate trial, were restricted to those with efficacy in the treatment of a major depressive episode with either major depressive or bipolar disorders, as supported by double-masked, placebo-controlled trials. For all participants, the classes of antidepressant treatments considered in determining the adequacy of trials included heterocyclics/tricyclics, monoamine oxidase inhibitors, selective serotonin reuptake inhibitors, bupropion, mirtazapine, nefazodone, trazodone, venlafaxine, reboxetine, and electroconvulsive therapy (ECT). In addition, for bipolar disorder, selected anticonvulsants (carbamazepine or lamotrigine) or lithium given at a sufficient oral dose or at sufficient serum concentration for an adequate duration could constitute an adequate trial. Thus, across unipolar and bipolar disorder, 11 classes of potentially adequate mood disorder treatments could count towards the study inclusion criteria. In addition, participants had to have shown no substantial response to at least 6 weeks of psychotherapy during any MDE.

\section{Study Overview}

The study was conducted at 21 sites. The Institutional Review Board at each site approved the study, which was performed under an Investigational Device Exemption (IDE) from the Food and Drug Administration (FDA) (see Acknowledgments). All study participants signed written informed consent forms.

The participants completed a pre-implantation baseline period (up to 45 days) during which clinical assessments were performed on two separate occasions. To qualify for device implantation, participants had to score $\geq 20$ (the average of the two baseline measurements) on the 24-item Hamilton Rating Scale for Depression $\left(\mathrm{HRSD}_{24}\right.$ ) (Hamilton 1960, 1967; Moberg et al 2001). Participants were not withdrawn from psychotropic medications. Rather, participants maintained a stable medication regimen for at least 4 weeks before the initial baseline visit, as well as during baseline, recovery, and the 10-week acute phase treatment period

Device implantation was followed by a 2-week, singlemasked recovery period during which the VNS device remained off to allow for surgical recovery. Participants were told that stimulation might or might not be turned on immediately after surgery. Many participants experience no sensations when the device is operated at low stimulus intensity, or even at higher intensities for some participants. These facts were communicated to participants to help preserve the blind.

The study was a double-masked trial with 1:1 randomization to sham VNS or active VNS. Those who received sham VNS were offered active VNS after this trial (Rush et al, this issue). A third party, independent from the investigators and any staff at the study sites, served as the randomization agent. Participant numbers were assigned in sequence for all study participants, regardless of the randomization assignment. The device programmer obtained the randomization assignment for each participant.

Participants had to score $\geq 18$ on the $\mathrm{HRSD}_{24}$ at visit 2 (14 days post-implantation) to enter the acute treatment phase. For the active VNS group, the device was turned on after the 2-week recovery period and the output current ( $\mathrm{mA}$ setting) was increased progressively to the maximal level that could be comfortably tolerated by the participant. Stimulus parameters were adjusted over the first 2 weeks (stimulation adjustment period) following the recovery period, and clinical assessments were performed weekly.

At 4 weeks post-implantation (i.e., after 2 weeks of stimulation initiation for the active VNS group), stimulation parameters were fixed for the remaining 8 weeks of the acute study. While a decrease in stimulation parameters was permitted if intolerable side effects developed, no participant required such adjustments. Participants were seen weekly for the next 2 weeks and then every other week over the ensuing 6 weeks. This fixed-dose stimulation period lasted 8 weeks. The total duration of stimulation was 10 weeks.

Identical procedures, including device implantation, were followed with the sham VNS group. In this group, however, the device was not turned on. The unmasked device programmer made all adjustments in stimulation parameters, including the sham adjustments. This person was not involved in any participant care or clinical assessments. The participants, outcome raters, and all other staff involved in clinical care and management were masked to whether or not VNS was active or sham. To preserve the mask to the raters, the unmasked programmer turned off the device at all clinic visits to prevent the occurrence of voice alterations should stimulation occur during the clinical assessment. The unmasked device programmer collected information on all adverse events, and, to further ensure masking, patients were scheduled to avoid overlap in the waiting room. 


\section{VNS Treatment}

The device implantation procedures and protocol-recommended treatment parameters were identical to those used in the studies for patients with pharmacoresistant epilepsy (Ben-Menachem et al 1994; Handforth et al 1998; Vagus Nerve Stimulation Study Group 1995) and in the open trial for patients with TRD (Sackeim et al 2001a). At each clinic visit, accuracy of stimulation parameters was verified, and the details of each stimulation occasion since the last visit were downloaded into a database stored on the VNS therapy system portable computer.

For the active VNS group, the protocol called for $20 \mathrm{~Hz}, 500$ $\mu s$ pulse width, and on/off cycle of $30 \mathrm{sec}$ on and 5 min off during the 2-week stimulation adjustment and acute phase trial period. The output current, beginning at $.25 \mathrm{~mA}$ as the lowest dose, was increased gradually (in $.25 \mathrm{~mA}$ increments) until a comfortable level was reached. Once this level was attained, participants left the clinic with the VNS device programmed at those settings. Additional increases (in $.25 \mathrm{~mA}$ steps) could be made up to $3.5 \mathrm{~mA}$ at any time during the stimulation adjustment period over the next 2 weeks.

\section{Concomitant Therapy}

Participants could be taking up to a total of five antidepressant, mood stabilizer, or other psychotropic medications (e.g., atypical antipsychotics), provided the medication type and dosage were kept stable throughout the baseline period and throughout the 12 weeks after implantation. Specifically, medication dosage could not be increased during this acute trial. The only psychotropic medication that could be added during the trial was trazodone (up to $300 \mathrm{mg} /$ day), for insomnia, as needed. Concomitant ECT, investigational drugs, or treatment with another investigational device was not permitted.

\section{Evaluations and Outcome Measurements}

Baseline evaluations included medical and psychiatric history, physical and neurological exams, and pre-surgical laboratory tests. Efficacy and safety data were gathered at the two baseline visits and at post-implantation weeks 1 and 2 (recovery period), weeks 3 and 4 (stimulation adjustment period), and weeks 5, 6, 8,10 , and 12 (fixed-dose stimulation period). Clinical assessments of depressive symptoms included the $\mathrm{HRSD}_{28}$ (Hamilton 1960, 1967; Moberg et al 2001) using a semistructured interview (Williams 1988) and the 10-item Montgomery-Äsberg Depression Rating Scale (MADRS) (Montgomery and Asberg 1979). Although the 28-item HRSD was administered to participants, the total of the first 24 questions was used to define the $\mathrm{HRSD}_{24}$ total score. Thus, $\mathrm{HRSD}_{24}$ results are reported for the study participants. The HRSD $_{24}$ includes the $\mathrm{HRSD}_{21}$ (Hamilton 1960, 1967) plus three additional items: 1) hopelessness, 2) helplessness, and 3) worthlessness. Thus, the $\mathrm{HRSD}_{24}$ includes the first 24 items of the $\mathrm{HRSD}_{28}$. The 30-item Inventory of Depressive Symptomatology Self-Report (IDS-SR 30 ) (Rush et al 1996, 2000a; Trivedi et al 2004; Rush et al, this issue) was used to measure self-reported depressive symptoms. Manic/hypomanic symptoms were rated by the Young Mania Rating Scale (YMRS) (Young et al 1978). The emergence of significant hypomania or mania was defined a priori as a score $\geq 15$ on the YMRS. In such cases, the DSM-IV criteria for mania were applied to declare the presence or absence of mania. The Clinical Global Severity (CGI-S) and Improvement (CGI-I) ratings (Guy 1976) were used to assess overall symptom severity and change. Functional outcomes (or quality of life) were assessed using the Medical Outcomes Study Short Form-36 (MOS SF-36) (Ware and Sherbourne 1992).
To assess inter-rater reliability, $\mathrm{HRSD}_{24}$ interviews were videotaped at key time points, including baseline and acute phase termination, and 6-, 12-, and 18-month follow-up. Over the course of the study, a random subset of these interviews were sent to the New York State Psychiatric Institute/Columbia University (New York, New York) for independent, time- and site-masked ratings. Expert clinical evaluators who were masked to treatment condition, time point in treatment or follow-up, and the $\mathrm{HRSD}_{24}$ scores obtained at the site, scored 379 of these interviews.

The $\mathrm{HRSD}_{24}$ assessments used a semi-structured interview guide adapted from Williams (1988) that provided a set of initial and follow-up questions for each item. The order of items was invariant and the descriptive anchors provided for each item addressed the ambiguities in the brief descriptions provided by the original $\mathrm{HRSD}_{24}$. Conventions for $\mathrm{HRSD}_{24}$ scoring were provided in the manual. To contribute ratings in the study, the clinical evaluators at each site had to pass a certification process. Certification involved achieving a strong level of reliability with consensus expert scores in rating a set of five practice tapes. Furthermore, each site rater was videotaped while using the semi-structured interview to conduct $\mathrm{HRSD}_{24}$ assessments of three patients. These tapes were evaluated for adequacy by expert clinical evaluators at the New York State Psychiatric Institute.

Adverse events and concomitant medications were coded using the COSTART (Coding Symbols for Thesaurus of Adverse Reaction Terms) (Food and Drug Administration 1995) dictionary.

\section{Statistical Methods}

Quintiles Inc. (Research Triangle Park, North Carolina), a clinical research organization (CRO), primarily conducted clinical monitoring visits at the 21 sites. Data were entered, verified, and analyzed using procedures that ensured the accuracy of the data and results. The primary outcome measure was the categorical classification of response, defined a priori as a $\geq 50 \%$ reduction after 10 weeks (LOCF) relative to the mean $\mathrm{HRSD}_{24}$ score obtained at the two baseline (pre-implantation) visits. The sample size was powered to detect a difference in the $\mathrm{HRSD}_{24}$ response rate of approximately $17 \%$. For secondary outcome measures, response was defined as a $\geq 50 \%$ reduction in the average of the two baseline scores for the MADRS or IDS-SR 30 , or a CGI-I score of 1 or 2 (much or very much improved). Evaluable participants in the sham and active VNS treatment groups were compared in response rates with Mantel-Haenszel chi-square tests stratifying by pooled investigator site. Treatment groups were also compared for average percent improvement from baseline in $\mathrm{HRSD}_{24}$, MADRS, IDS-SR 30 , SF-36, and YMRS scores via analyses of covariance (ANCOVA) adjusting for baseline value and pooled investigator site. A repeated measures linear regression analysis was used to compare differences in outcome between the sham group and the active group. Demographic and clinical features of the sham VNS group were compared with those of the active VNS group with ANOVAs adjusting for pooled investigator site, and Mantel-Haenszel chi-square tests stratified by pooled investigator site, or Fisher's exact test, as appropriate. Sites were pooled prior to unblinding into four groups for statistical adjustment, such that sites enrolling a similar number of participants were combined in the same pooled site. Statistically significant $p$-values were considered to be $\leq .050$. Inferential conclusions regarding VNS effectiveness in this study were limited to the single a priori primary outcome $\left(\mathrm{HRSD}_{24}\right.$ re- 
Table 1. Baseline Demographics and Clinical Features

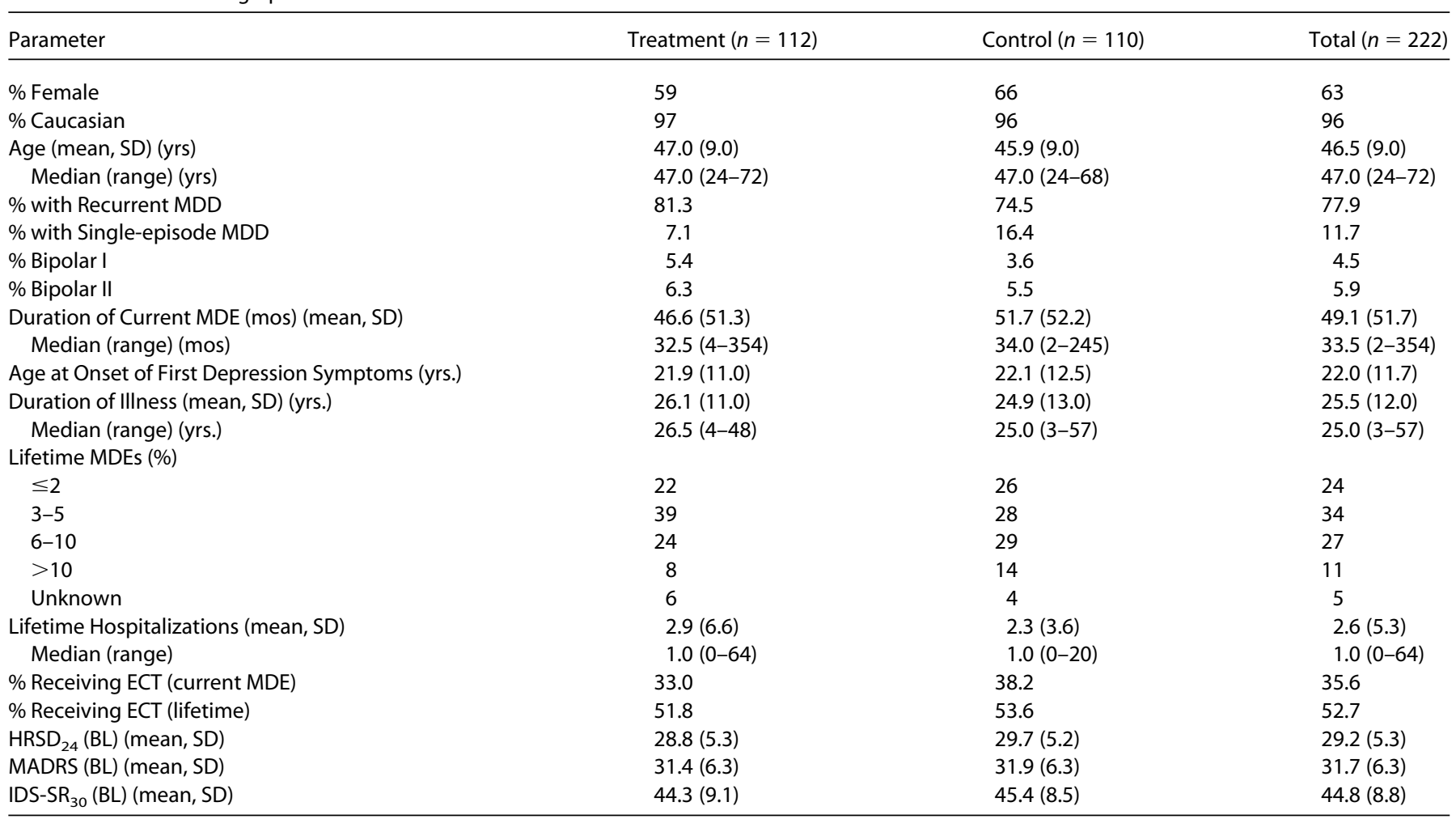

$M D D$, major depressive disorder; $M D E$, major depressive episode; $E C T$, electroconvulsive therapy; $\mathrm{HRSD}_{24}$, 24-item Hamilton Rating Scale For Depression; BL, ; MADRS, Montgomery-Åsberg Depression Rating Scale; IDS-SR ${ }_{30}$, Inventory of Depressive Symptomatology-Self-Report.

sponse). We interpreted all $p$-values from secondary outcomes as descriptive in nature; no adjustments were made for multiple comparisons.

\section{Results}

\section{Sample Characteristics}

Of the 266 participants enrolled in the trial, 235 participants were implanted. Altogether, 21 sites enrolled from four to 22 participants (mean 12.7; median 13), with only five sites enrolling fewer than 10 participants. Thirty-one enrolled participants were discontinued before implantation because they did not meet eligibility criteria $(n=13)$ or withdrew consent $(n=18)$. Of 235 implanted participants, 13 participants were not evaluable for efficacy: four did not meet eligibility criteria for continuing in the acute trial after implantation (their $\mathrm{HRSD}_{24}$ scores were $<18$ ), and nine had protocol violations after randomization (e.g., medication additions in violation of the requirement for a stable medication regimen). Thus, 222 participants are included in the efficacy analysis, with 112 participants randomized to the active VNS group and 110 randomized to the sham VNS group. Demographic data are reported on the 222 evaluable participants, and safety findings are reported on the total 235 implanted participants. Only three of the 235 participants (all in the VNS active stimulation group) withdrew from the study because of AEs, and one of the participants who withdrew did so at the end of the study. Table 1 presents the clinical and demographic features of the evaluable sample $(n=222)$. No statistically significant differences were noted in the clinical and demographic features between the active and the sham groups.

On average, participants in both groups had received over 16 unique mood disorder treatments before they entered the study, and had tried more than nine such medications during the current MDE. From a lifetime perspective, $89 \%$ had been treated with fluoxetine, $89 \%$ with bupropion, and $88 \%$ with venlafaxine. Prior treatments (lifetime and current MDE) were similar for the active VNS and sham VNS groups. Over half (53\%) of the evaluable sample had a history of ECT, and 36\% had received ECT during the current MDE.

Table 2 shows the distribution of ATHF-defined adequate treatment trials in the current MDE. The active and sham VNS groups did not differ in the mean and distributions of the number of adequate trials during the current $\operatorname{MDE}(p=.5020$, CochranMantel-Haenszel).

Table 3 summarizes the treatments being taken in addition to VNS during the acute phase trial. The minor differences in the frequency of use of any of the concomitant treatments between the active and sham groups were not clinically meaningful.

Table 2. Percent of Participants with Various Degrees of Treatment Resistance

\begin{tabular}{cccc}
\hline $\begin{array}{c}\text { Number of Unsuccessful } \\
\text { ATHF Treatments }^{a}\end{array}$ & $\begin{array}{c}\text { Treatment } \\
(n=112)\end{array}$ & $\begin{array}{c}\text { Control } \\
(n=110)\end{array}$ & $\begin{array}{c}\text { Total } \\
(n=222)\end{array}$ \\
\hline 2 & $30.4 \%$ & $31.8 \%$ & $31.1 \%$ \\
3 & $23.2 \%$ & $28.2 \%$ & $25.7 \%$ \\
4 & $20.5 \%$ & $18.2 \%$ & $19.4 \%$ \\
5 & $17.0 \%$ & $12.7 \%$ & $14.9 \%$ \\
$6^{b}$ & $9.0 \%$ & $9.1 \%$ & $9.0 \%$ \\
\hline
\end{tabular}

ATHF, Antidepressant Treatment History Form.

${ }^{a}$ Unsuccessful ATHF treatment refers only to treatments rated on ATHF for the current major depressive episode.

${ }^{b}$ One participant in the treatment group had failed 7 ATHF treatments. 
Table 3. Concomitant Mood Disorder Treatments During the Acute Phase Trial $^{a}$

\begin{tabular}{lccc}
\hline Category of Treatment & $\begin{array}{c}\text { Treatment } \\
(n=112)\end{array}$ & $\begin{array}{c}\text { Control } \\
(n=110)\end{array}$ & $\begin{array}{c}\text { Total } \\
(n=222)\end{array}$ \\
\hline Heterocyclics/TCAs & $10 \%$ & $14 \%$ & $12 \%$ \\
SSRIs & $46 \%$ & $44 \%$ & $45 \%$ \\
MAOls & $6 \%$ & $7 \%$ & $6 \%$ \\
Anticonvulsants-total & $30 \%$ & $33 \%$ & $31 \%$ \\
$\quad$ (carbamazepine, lamotrigine, & & & \\
$\quad$ valproic acid, gabapentin, & & & \\
$\quad$ topiramate, tiagabine) & $20 \%$ & $22 \%$ & $21 \%$ \\
Stimulants & $26 \%$ & $24 \%$ & $25 \%$ \\
Atypical Antipsychotics & $0 \%$ & $6 \%$ & $3 \%$ \\
Non atypical Antipsychotics & $65 \%$ & $50 \%$ & $57 \%$ \\
Anxiolytics & & & \\
Specific Medications of Interest & $27 \%$ & $18 \%$ & $22 \%$ \\
$\quad$ Bupropion & $26 \%$ & $29 \%$ & $27 \%$ \\
$\quad$ Venlafaxine & $15 \%$ & $11 \%$ & $13 \%$ \\
$\quad$ Mirtazapine & $14 \%$ & $6 \%$ & $10 \%$ \\
$\quad$ Nefazodone & $17 \%$ & $14 \%$ & $16 \%$ \\
$\quad$ Trazodone & $15 \%$ & $7 \%$ & $11 \%$ \\
$\quad$ Lithium &
\end{tabular}

TCA, tricyclic antidepressant; SSRI, selective serotonin reuptake inhibitor; $\mathrm{MAOl}$, monoamine oxidase inhibitor.

${ }^{a}$ The differences between the treatment and control groups were not found to be statistically significant for categories of treatment $\left(\chi^{2}=9.40 ; d f\right.$ $=7)$ or specific medications of interest $\left(\chi^{2}=5.49 ; d f=5\right)$.

\section{Efficacy}

VNS Parameters. Median output current at the last recorded visit was $.75 \mathrm{~mA}$ (range, .00 to $1.50 \mathrm{~mA}$ ) and the average output current was $.67 \mathrm{~mA}(\mathrm{SD}=.33)$. Of the 111 evaluable participants with values recorded at study exit, $53(48 \%)$ received $\leq .50 \mathrm{~mA}$ and $58(52 \%)$ received $>.50 \mathrm{~mA}$ output current. Median signal frequency was $20 \mathrm{~Hz}$ (range, 10 to $20 \mathrm{~Hz}$ ), median pulse width was $500 \mu \mathrm{sec}$ (range, 130 to $500 \mu \mathrm{sec}$ ) median on time was $30 \mathrm{sec}$ (range, 14 to $30 \mathrm{sec}$ ), and off time was $5 \mathrm{~min}$. For the $\mathrm{HRSD}_{24}$ scores, the intraclass correlation coefficent (ICC, single rater) across the 379 interviews was .94, indicating high inter-rater reliability for the $\mathrm{HRSD}_{24}$ scores. No site showed poor reliability as the ICC value computed per site ranged from .87 to .98 , with a median of .95 . The average discrepancy between the original ratings at each site and those of the Columbia University rater, while statistically significant $(d f=378, t=5.08, p<.001)$, was slight and reflected the original raters scoring the patients .78 points higher on the $\mathrm{HRSD}_{24}$ than the Columbia rater (Original rater: $23.90 \pm 8.74$; Columbia rater: $23.12 \pm 8.33$ ). The magnitude of the discrepancies between the two sets of raters (both directional and absolute) did not differ among the sites.

Table 4 summarizes the LOCF outcomes for the efficacy evaluable sample $(n=222)$. Response rates were generally similar across sites, although some variation was seen (seven sites had $<10 \%$ response rate, four sites had $\geq 25 \%$ response rate). Figure 1 shows the response rates by $\mathrm{HRSD}_{24}$ and IDS-SR 30 using the LOCF sample. The difference in the $\mathrm{HRSD}_{24}$ response rates was not significant (chi-square $=1.32, d f=1, p=.251$ ), but the difference in the IDS-SR 30 response rates was statistically significant (chi-square $=4.62, d f=1, p=.032$ ). Two other secondary measures of efficacy were not statistically significant, the MADRS (chi-square $=.778, d f=1, p=.378$ ) and the CGI-I (chi-square $=.208, d f=1, p=.648$ ).

A repeated measures linear regression analysis was performed for the evaluable sample for raw scores of the active
Table 4. Clinical Outcomes for VNS versus Sham (at 10 weeks LOCF)

\begin{tabular}{|c|c|c|c|}
\hline Rating & $\begin{array}{c}\text { Treatment } \\
(n=112)\end{array}$ & $\begin{array}{c}\text { Control } \\
(n=110)\end{array}$ & $p$ \\
\hline $\mathrm{HRSD}_{24}$ Response Rate (\%) & $15.2 \%$ & $10.0 \%$ & $.25^{a}$ \\
\hline MADRS Response Rate $(\%)^{c}$ & $15.2 \%$ & $11.0 \%$ & .378 \\
\hline CGI-I Response Rate $(\%)^{d}$ & $13.9 \%$ & $11.8 \%$ & .648 \\
\hline IDS-SR ${ }_{30}$ Response Rate (\%) ${ }^{e}$ & $17.0 \%$ & $7.3 \%$ & $.032^{b}$ \\
\hline \multicolumn{4}{|l|}{$\mathrm{HRSD}_{24} \%$ Improvement from } \\
\hline Baseline (mean, SD) & $16.3 \pm 28.1$ & $15.3 \pm 25.5$ & .639 \\
\hline \multicolumn{4}{|l|}{ MADRS \% Improvement from } \\
\hline Baseline (mean, SD) & $17.1 \pm 31.2$ & $12.4 \pm 27.1$ & .208 \\
\hline \multicolumn{4}{|l|}{ IDS-SR $30 \%$ Improvement from } \\
\hline Baseline (mean, SD) & $21.2 \pm 25.4$ & $16.3 \pm 26.2$ & .158 \\
\hline
\end{tabular}

VNS, vagus nerve stimulation; LOCF, last observation carried forward; $\mathrm{HRSD}_{24}$, 24-item Hamilton Rating Scale for Depression; MADRS, Montgomery-Åsberg Depression Rating Scale; CGI-I, Clinical Global Improvement ratings; IDS-SR 30 , 30 item Inventory of Depressive Symptomalogy-Self-Report.

aPrimary outcome.

${ }^{b}$ Statistically significant secondary parameter.

'One patient in the control group did not have a MADRS assessment completed during the study.

${ }^{d}$ One patient in the treatment group and one patient in the control group did not have a CGl assessment completed during the study.

eOne patient in the control group did not have an IDS-SR ${ }_{30}$ assessment completed during the study.

versus sham groups. At 12 weeks, the estimated difference for the $\mathrm{HRSD}_{24}$ was -.769 , SE .80, 95\% CI $(-2.34, .80), p=.336$ and the estimated difference for the IDS-SR was -2.374 , SE $1.23,95 \%$ CI $(-4.78, .03), p=.053$.

The active and sham VNS groups did not differ on either physical or mental component of the MOS-SF36. For the physical component, mean change was -.9 (SD $=8.3)$ for the VNS group $(n=107)$ and $-1.6(\mathrm{SD}=8.4)$ for the sham group $(n=107 ; F=$ $.50, d f=[1,208], p=.480$, ANCOVA). For the mental component, mean change was $5.0(\mathrm{SD}=11.6)$ for the VNS group $(n=$ $107)$ and $4.0(\mathrm{SD}=10.2)$ for the sham group $(n=107 ; F=.69$, $d f=[1,208], p=.406$, ANCOVA).

\section{Adverse Events}

Two participants, both in the active VNS group, did not complete the acute study because of adverse events (AEs). One

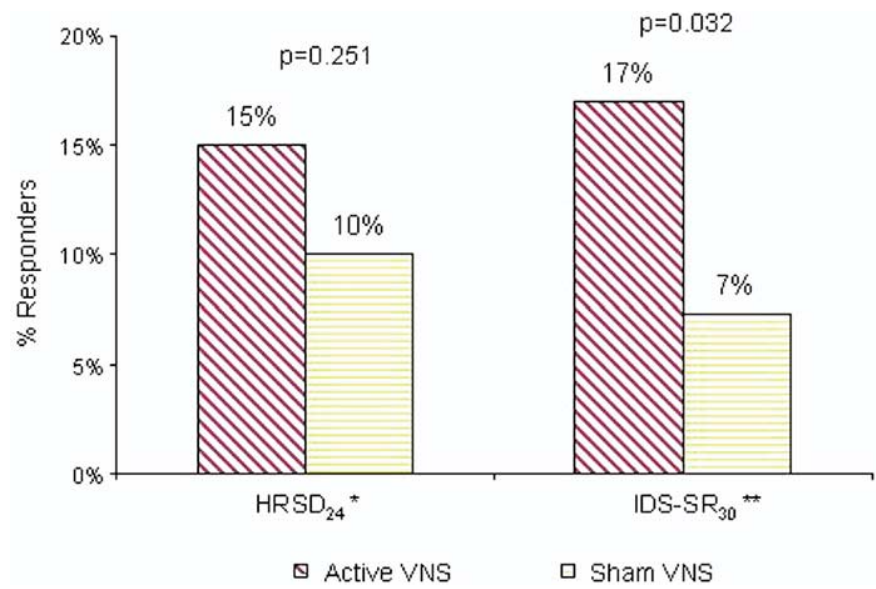

Figure 1. Between Group last observation carried forward (LOCF) Responder Rates ( $\mathrm{HRSD}_{24}$ and IDS-SR $\left.{ }_{30}\right)(n=222)$. $\mathrm{HRSD}_{24}$, 24-item Hamilton Rating Scale for Depression; IDS-SR ${ }_{30}$, Inventory of Depressive Symptomatology - Self-Report; VNS, vagus nerve stimulation. ${ }^{*}$ Primary outcome; ** Secondary outcome. 
participant underwent device explantation due to infection. The second participant committed suicide after 5 weeks of VNS treatment, which, in the opinion of the investigator, was most likely due to the underlying mood disorder and not study participation. At the end of the study, a third participant withdrew because of hoarseness.

Overall (Table 5), the AEs encountered were similar to those of the prior study of VNS for treatment-resistant depression (TRD) (Sackeim et al 2001b) and for epilepsy (Morris and Mueller 1999). The most common AE was voice alteration. Of 30 total serious adverse events (SAEs) involving 27 participants, 16 SAEs occurred in the active VNS group and 14 in the sham group. This total included 12 SAEs involving 11 participants of worsened depression that required hospitalization (seven participants in sham, four participants in active VNS, and one participant who had not yet received stimulation, but who was assigned to the active VNS group). One case of asystole and one case of bradycardia, both during surgery, occurred in the active VNS group, while neither occurred in the sham group. No other cardiac SAEs occurred during study treatment. The randomized groups had no meaningful differences in change from baseline for temperature, heart rate, diastolic or systolic blood pressure, or in weight (active VNS lost an average of $1.2 \pm 8.0 \mathrm{lb}$; sham VNS lost an average of $2.1 \pm 8.7 \mathrm{lb}$ ).

Two participants in the active VNS group (one of whom had a diagnosis of bipolar I disorder at baseline) met the threshold of significant hypomania or mania, a score $\geq 15$ on the YMRS, which was validated by DSM-IV criteria. Neither participant underwent VNS dose reduction or discontinued VNS. These elevated YMRS scores occurred at initiation of stimulation for one participant and 8 weeks of VNS for the other. Both resolved spontaneously after 1 to 2 weeks.

\section{Discussion}

This trial is the first sham-controlled, acute-treatment study to be conducted in such a chronically ill, treatment-resistant depressed population characterized by high rates of prior ECT (53\%), prior hospitalization (63\%), and substantial disability (SF-36 Mental Score of approximately 27 out of a possible 100 points). Depressed patients seen in general practice scored 46.3 in the Medical Outcomes Study (Komaroff et al 1996). This study did not yield definitive evidence of short-term efficacy in the context of this chronically ill, treatment-resistant, depressed

Table 5. Percent of Participants $(n=235)$ Reporting Adverse Events ${ }^{a}$

\begin{tabular}{lcc}
\hline Adverse Event & $\begin{array}{c}\text { VNS Group } \\
(n=119)\end{array}$ & $\begin{array}{c}\text { Sham Control Group } \\
(n=116)\end{array}$ \\
\hline Voice Alteration & $68 \%$ & $38 \%$ \\
Cough Increased & $29 \%$ & $9 \%$ \\
Dyspnea & $23 \%$ & $14 \%$ \\
Dysphagia & $21 \%$ & $11 \%$ \\
Neck Pain & $21 \%$ & $10 \%$ \\
Paresthesia & $16 \%$ & $10 \%$ \\
Vomiting & $11 \%$ & $5 \%$ \\
Laryngismus & $11 \%$ & $2 \%$ \\
Dyspepsia & $10 \%$ & $5 \%$ \\
Wound Infection & $8 \%$ & $2 \%$ \\
Palpitations & $5 \%$ & $3 \%$ \\
\hline
\end{tabular}

VNS, vagus nerve stimulation.

${ }^{a}$ All adverse events occurring in $\geq 5 \%$ of participants receiving stimulation and $1.5 \times$ sham control group are listed whether or not related to stimulation or implantation. population. This trial revealed that A) VNS was well tolerated; B) the adverse event profile and $\mathrm{AE}$ rates closely approximated those seen in patients with epilepsy; C) the modest difference in response rates to active VNS (15.2\%), and sham VNS group $(10.0 \%)$ was not statistically significant for the $\mathrm{HRSD}_{24}$, the primary measure, and D) the secondary measure, the IDS-SR 30 revealed a significant difference favoring VNS over sham (analyzed without correction for multiple tests). Importantly, only a $10 \%$ placebo (sham) response was found in this rarely studied population. These findings indicate either that VNS is ineffective (at least in the short run), or that this study could be a failed trial because of a variety of issues including: (1) study design, (2) study sample, and (3) treatment delivery.

\section{Study Design}

The sample size was powered to detect a difference of approximately $17 \%$ between placebo response and treatment response. The $\mathrm{HRSD}_{24}$ response rate was $15.2 \%$ (17/112, LOCF) for participants in the active VNS group and $10.0 \%(11 / 110)$ for those in the sham group. We could have simply underpowered the study, having assumed a better response rate than was obtained after the 10 weeks of VNS.

Although 10- to 12-week acute studies for regulatory approval are common for studies of depression, studies of TRD are less common, and longer acute-phase studies may be necessary in TRD populations (Rush et al 2003b). Naturalistic follow-up studies of both VNS-treated epilepsy patients (DeGiorgio et al 2000; Morris and Mueller 1999) and TRD patients (Marangell et al 2002) suggest that the effect of VNS may increase after the acute phase. If more time is needed for VNS benefits to become evident in this TRD group, a longer randomized controlled trial might have produced positive findings as suggested by the longer-term, follow-up data on this cohort (Rush et al, this issue), as well as a comparison with a benchmark control group (George et al, this issue).

Only 25 of the 235 enrolled participants were classified as bipolar I or II. Of the 222 participants in the evaluable LOCF sample, 23 participants were classified as bipolar. These participants with bipolar disorder had a response rate of $8.7 \%(2 / 23)$ as compared with those with MDD $13.1 \%(26 / 199)$ as graded by the $\mathrm{HRSD}_{24}$. Thus, inclusion of bipolar depressed participants did not likely affect results.

\section{Study Sample}

The study design was nearly identical to the Sackeim et al (2001b) design, except that the degree of allowable treatment resistance was capped at $\leq 6$ antidepressant trials from different classes during the current MDE. However, the overall mean duration of illness (time from the onset of the first mood episode to study entry) was greater in the present study than for the Sackeim et al (2001b) group (25.5 vs. 18.1 years), and the present study had fewer participants with bipolar disorder (10.4\% versus 27.0\%). The duration of the current episode in the present study was much shorter ( 4.1 years versus 9.9 years; median 2.8 years vs. 6.6 years) than the Sackeim et al (2001b) group.

These data argue that the degree of lifetime treatment resistance among participants in the present study was likely not lower than for the Sackeim et al (2001b) study, and was potentially higher. Because greater treatment resistance is associated with lower acute response rates in ECT (Sackeim et al 1990), in mood stabilizers among patients with bipolar disorder (Barbee and Jamhour 2002), and in VNS (Sackeim et al 2001b), a 
potentially higher treatment resistance would be expected to reduce response rates for 10 weeks of VNS.

\section{Treatment Delivery}

Although no direct evidence links the amount of current delivered during VNS with the outcome in depression, the average output current in the epilepsy studies was $1.3 \mathrm{~mA}$ for patients who received active VNS (Handforth et al 1998). In Sackeim et al (2001b), the median output current was $.75 \mathrm{~mA}$ (range, $.25 \mathrm{~mA}$ to $3.0 \mathrm{~mA})$, and the average was $.96 \mathrm{~mA}(\mathrm{SD}=$ $.54 \mathrm{~mA}$ ). In the present study, the median output current at the last recorded visit was $.75 \mathrm{~mA}$ (range, .00 to $1.50 \mathrm{~mA}$ ) and the average output current was $.67 \mathrm{~mA}(\mathrm{SD}=.33)$. Of the 111 evaluable participants with values recorded at study exit, 53 (48\%) received $\leq .50 \mathrm{~mA}$ and $58(52 \%)$ received $>.50 \mathrm{~mA}$ output current. The use of lower output current settings in this study could be attributed to several factors. Data from the Sackeim et al (2001b) study did not indicate that higher output currents were associated with a better response. Thus, the need to push the output current to the maximally tolerated dose was not established at the time of this study, and, indeed, it may not be necessary. The need to maintain the blind was not a factor in the Sackeim et al (2001b) trial. However, in the present study, the desire to protect the blind may have limited the number of opportunities that were provided to adjust the dose for tolerability. An exploratory analysis of this acute study found no relationship between output current and the percentage of change in the $\mathrm{HRSD}_{24}$. Theoretically, the lower output current settings used in this study could have limited the acute response rates, though the actual differences in dosage are modest. Furthermore, the current study had similar rates of adverse events as reported in the Sackeim et al (2001b) study, suggesting that the VNS dose (current) was pushed to at least the same degree of tolerability as in the Sackeim et al (2001b) study.

In sum, VNS was safe and well tolerated. This study did not yield definitive evidence of short-term efficacy for adjunctive VNS in treatment-resistant depression. By all measures, VNS was associated with greater symptom reduction. In the primary analysis, $\mathrm{HRSD}_{24}$, the response rate was $15.2 \%$ in the active treatment group and $10.0 \%$ in the sham group, a modest difference that was not statistically significant. A secondary outcome, the IDS-SR ${ }_{30}$, which revealed a significantly greater response rate with VNS (17.0\%) than with sham (7.3\%), was not corrected for multiple comparisons. Whether study design, study sample, or stimulation parameters contributed to this failed acute-phase trial is unclear. Alternatively, this acute phase trial may simply have failed because VNS is not effective in reducing the symptoms of depression. Given the 25-year history of depression and substantial degree of treatment resistance of these participants, longer treatment with VNS may be necessary to achieve clinically meaningful benefit (Rush et al, this issue).

This study was supported by Cyberonics, Inc., through contracts to investigating sites. Statistical analyses were conducted by Quintiles Inc. and reviewed by the senior authors. The following principal investigators and sites participated in this study (the approving IRBs are in parentheses): J. Barry, M.D., Stanford University School of Medicine (Stanford University/ Human Subjects in Medical Research); M. Burke, M.D., Psychiatric Research Institute (Via Christi Regional Medical Center Institutional Review Board); W. Burke, University of Nebraska Medical Center (University of Nebraska Institutional Review
Board); L. Carpenter, M.D., Brown University/Butler Hospital (Butler Hospital IRB); C. Conway, M.D., St. Louis University Health Science Center (St. Louis University IRB); R. Cooke, M.D., University of Toronto/Centre for Addiction and Mental Health (Centre for Addiction and Mental Health Research Ethics Board); R.A. Dominguez, M.D., University of Miami School of Medicine (University of Miami School of Medicine/Human Subjects Research Office); D. Dunner, M.D., University of Washington Center for Anxiety and Depression (Human Subjects Division, University of Washington); M.S. George, M.D., Medical University of South Carolina (Office of Research Integrity IRB \#3); D. Ginsberg, M.D., New York University Medical Center (NYU Medical Center Institutional Board of Research Associates); R. Howland, M.D., University of Pittsburgh School of Medicine (University of Pittsburgh Institutional Review Board); M. Husain, M.D., The University of Texas Southwestern Medical Center (Office of Institutional Review Board UT Southwestern Medical Center); M. Kling, M.D., University of Maryland (University of Maryland Baltimore Institutional Review Board); L.B. Marangell, M.D., Baylor College of Medicine (Baylor College of Medicine IRB); F. Moreno, M.D., University of Arizona Health Science Center (Human Subjects Protection Program); A. Nierenberg, M.D., Massachusetts General Hospital (Massachusetts General Hospital IRB); P. Ninan, M.D., Emory University School of Medicine (Emory University Institutional Review Board); B. Rittberg, M.D., University of Minnesota Medical School (Research Subjects' Protection Program IRB); T. Schwartz, M.D., SUNY Upstate Medical University at Syracuse (State University of New York Upstate Medical University Institutional Review Board); $M$. Soliman, M.D., University of California San Diego Department of Psychiatry (UCSD Human Research Protection Program); J. Zajecka, M.D., Rush Presbyterian-St. Luke's Medical Center (Rush University Medical Center IRB).

We declare the following financial relationships with Cyberonics, Inc., during the past five years: John Rush, M.D. Speaker's Bureau, research support, consultant; Lauren $B$. Marangell, M.D. - Speaker's Bureau, research support, consultant; Harold A. Sackeim, Ph.D. Speaker's Bureau, research support, consultant; Mark S. George, M.D. Speaker's Bureau, research support, consultant; Stephen K. Brannan M.D. - employee and stockholder; Sonia M. Davis, Dr.P.H. - consultant; employee of Quintiles; Robert Howland, M.D. - Speaker's Bureau, research support; Mitchel A. Kling, M.D. - Speaker's Bureau, research support, consultant; Barry R. Rittberg, M.D.Speaker's Bureau, research support; William J. Burke, M.D. research support, consultant; Mark H. Rapaport, M.D. - Speaker's Bureau, research support, consultant; John Zajecka, M.D. Speaker's Bureau, research support; Andrew A. Nierenberg, M.D. - Speaker's Bureau, research support; Mustafa M. Husain, M.D. Speaker's Bureau, research support; David Ginsberg, M.D. Speaker's Bureau, research support, consultant; Robert G. Cooke, M.D. - research support

American Psychiatric Association (2000): Diagnostic and statistical manual of mental disorders - DSM-IV-TR, 4th ed. Washington, DC: American Psychiatric Association.

Barbee JG, Jamhour NJ (2002): Lamotrigine as an augmentation agent in treatment-resistant depression. J Clin Psychiatry 63:737-741.

Ben-Menachem E, Hamberger A, Hedner T, Hammond EJ, Uthman BM, Slater $J$, et al (1995): Effects of vagus nerve stimulation on amino acids and other metabolites in the CSF of patients with partial seizures. Epilepsy Res 20:221-227.

Ben-Menachem E, ManonEspaillat R, Ristanovic R, Wilder BJ, Stefan H, Mirza $W$, et al (1994): Vagus nerve stimulation for treatment of partial seizures: 
1 A controlled study of effect on seizures. First International Vagus Nerve Stimulation Study Group. Epilepsia 35:616-626.

Carpenter LL, Moreno FA, Kling MA, Anderson GM, Regenold WT, Labiner DM, Price LH (2004): Effect of vagus nerve stimulation on cerebrospinal fluid monoamine metabolites, norepinephrine, and gammaaminobutyric acid concentrations in depressed patients. Biol Psychiatry $56: 418-426$

Chae JH, Nahas Z, Lomarev M, Denslow S, Lorberbaum JP, Bohning DE, et al (2003): A review of functional neuroimaging studies of vagus nerve stimulation (VNS). J Psychiatr Res 37:443-455.

DeGiorgio CM, Schachter SC, Handforth A, Salinsky M, Thompson J, Uthman $B$, et al (2000): Prospective long-term study of vagus nerve stimulation for the treatment of refractory seizures. Epilepsia 41:1195-1200.

Elger G, Hoppe C, Falkai P, Rush AJ, Elger CE (2000): Vagus nerve stimulation is associated with mood improvements in epilepsy patients. Epilepsy Res 42:203-210.

Food and Drug Administration (1995): COSTART: Coding Symbols for Thesaurus of Adverse Reaction Terms, $5^{\text {th }}$ ed. Rockville, MD: US Department of Health and Human Services, Center for Drug Evaluation and Research, Food and Drug Administration, Center for Drugs and Biologics.

George MS, Sackeim HA, Marangell LB, Husain MM, Nahas Z, Lisanby SH, et al (2000): Vagus nerve stimulation. A potential therapy for resistant depression?. Psychiatr Clin North Am 23:757-783.

Guy W (1976): ECDEU. Assessment Manual for Psychopharmacology. Publication No. 76-338. Washington, D.C.: Superintendent of Documents, U.S. Government Printing Office, U.S. Department of Health, Education, and Welfare.

Hamilton M (1960): A rating scale for depression. J Neurol Neurosurg Psychiatry 23:56-62.

Hamilton M (1967): Development of a rating scale for primary depressive illness. Br J Soc Clin Psychol 6:278-296.

Hammond EJ, Uthman BM, Reid SA, Wilder BJ (1992): Electrophysiologic studies of cervical vagus nerve stimulation in humans: II Evoked potentials. Epilepsia 33:1021-1028.

Handforth A, DeGiorgio CM, Schachter SC, Uthman BM, Naritoku DK, Tecoma ES, et al (1998): Vagus nerve stimulation therapy for partialonset seizures: a randomized active-control trial. Neurology 51:48-55.

Harden CL, Pulver MC, Ravdin LD, Nikolov B, Halper JP, Labar DR (2000): A Pilot Study of Mood in Epilepsy Patients Treated with Vagus Nerve Stimulation. Epilepsy Behav 1:93-99.

Henry TR, Bakay RA, Votaw JR, Pennell PB, Epstein CM, Faber TL, et al (1998): Brain blood flow alterations induced by therapeutic vagus nerve stimulation in partial epilepsy: I. Acute effects at high and low levels of stimulation. Epilepsia 39:983-990.

Komaroff AL, Fagioli LR, Doolittle TH, Gandek B, Gleit MA, Guerriero RT, et al (1996): Health status in patients with chronic fatigue syndrome and in general population and disease comparison groups. Am J Med 101:281-290.

Marangell LB, Rush AJ, George MS, Sackeim HA, Johnson CR, Husain MM, et al (2002): Vagus nerve stimulation (VNS) for major depressive episodes: one year outcomes. Biol Psychiatry 51:280-287.

Moberg PJ, Lazarus LW, Mesholam RI, Bilker W, Chuy IL, Neyman I, Markvart $V$ (2001): Comparison of the standard and structured interview guide for the Hamilton Depression Rating Scale in depressed geriatric inpatients. Am J Geriatr Psychiatry 9:35-40.

Montgomery SA, Asberg M (1979): A new depression scale designed to be sensitive to change. Br J Psychiatry 134:382-389.
Morris GL 3rd, Mueller WM (1999): Long-term treatment with vagus nerve stimulation in patients with refractory epilepsy. The Vagus Nerve Stimulation Study Group E01-E05. Neurology 53:1731-1735.

Prudic J, Haskett RF, Mulsant B, Malone KM, Pettinati HM, Stephens S, et al (1996): Resistance to antidepressant medications and short-term clinical response to ECT. Am J Psychiatry 153:985-992.

Prudic J, Sackeim HA, Devanand DP (1990): Medication resistance and clinical response to electroconvulsive therapy. Psychiatry Res 31:287-296.

Rush AJ, Carmody TJ, Reimitz PE (2000a): The Inventory of Depressive Symptomatology (IDS): clinician (IDS-C) and self-report (IDS-SR) ratings of depressive symptoms. Int J Methods Psychiatr Res 9:45-59.

Rush AJ, George MS, Sackeim HA, Marangell LB, Husain M, Nahas Z, et al (2003a): Continuing benefit of VNS therapy over 2 years for treatmentresistant depression [Poster]. Presented at the 43rd Annual NCDEU Annual Meeting Boca Raton, Florida.

Rush AJ, George MS, Sackeim HA, Marangell LB, Husain MM, Giller C, et al (2000b): Vagus nerve stimulation (VNS) for treatment-resistant depressions: a multicenter study. Biol Psychiatry 47:276-286.

Rush AJ, Gullion CM, Basco MR, Jarrett RB, Trivedi MH (1996): The Inventory of Depressive Symptomatology (IDS): psychometric properties. Psychol Med 26:477-486.

Rush AJ, Thase ME, Dube S (2003b): Research issues in the study of difficultto-treat depression. Biol Psychiatry 53:743-753.

Sackeim HA (2001): The definition and meaning of treatment-resistant depression. J Clin Psychiatry 62:11-17.

Sackeim HA, Keilp JG, Rush AJ, George MS, Marangell LB, Dormer JS, et al (2001a): The effects of vagus nerve stimulation on cognitive performance in patients with treatment-resistant depression. Neuropsychiatry Neuropsychol Behav Neurol 14:53-62.

Sackeim HA, Prudic J, Devanand DP, Decina P, Kerr B, Malitz S (1990): The impact of medication resistance and continuation pharmacotherapy on relapse following response to electroconvulsive therapy in major depression. J Clin Psychopharmacol 10:96-104.

Sackeim HA, Rush AJ, George MS, Marangell LB, Husain MM, Nahas Z, et al (2001b): Vagus nerve stimulation (VNS) for treatment-resistant depression: efficacy, side effects, and predictors of outcome. Neuropsychopharmacology 25:713-728.

Schachter SC, Saper CB (1998): Vagus nerve stimulation. Epilepsia 39:677686.

Trivedi MH, Rush AJ, Ibrahim HM, Carmody TJ, Biggs MM, Suppes T, et al (2004): The Inventory of Depressive Symptomatology, Clinician Rating (IDS-C) and Self-Report (IDS-SR), and the Quick Inventory of Depressive Symptomatology, Clinician Rating (QIDS-C) and Self-Report (QIDS-SR) in public sector patients with mood disorders: a psychometric evaluation. Psychol Med 34:73-82.

Vagus Nerve Stimulation Study Group (1995): A randomized controlled trial of chronic vagus nerve stimulation for treatment of medically intractable seizures. Neurology 45:224-230.

Ware JE, Sherbourne CD (1992): The MOS 36-item short-form health survey (SF-36). I. Conceptual framework and item selection. Med Care 30:473483.

Williams JB (1988): A structured interview guide for the Hamilton Depression Rating Scale. Arch Gen Psychiatry 45:742-747.

Young RC, Biggs JT, Ziegler VE, Meyer DA (1978): A rating scale for mania: reliability, validity and sensitivity. Br J Psychiatry 133:429-435. 